\title{
Obtaining and managing data sets for individual participant data meta-analysis: scoping review and practical guide
}

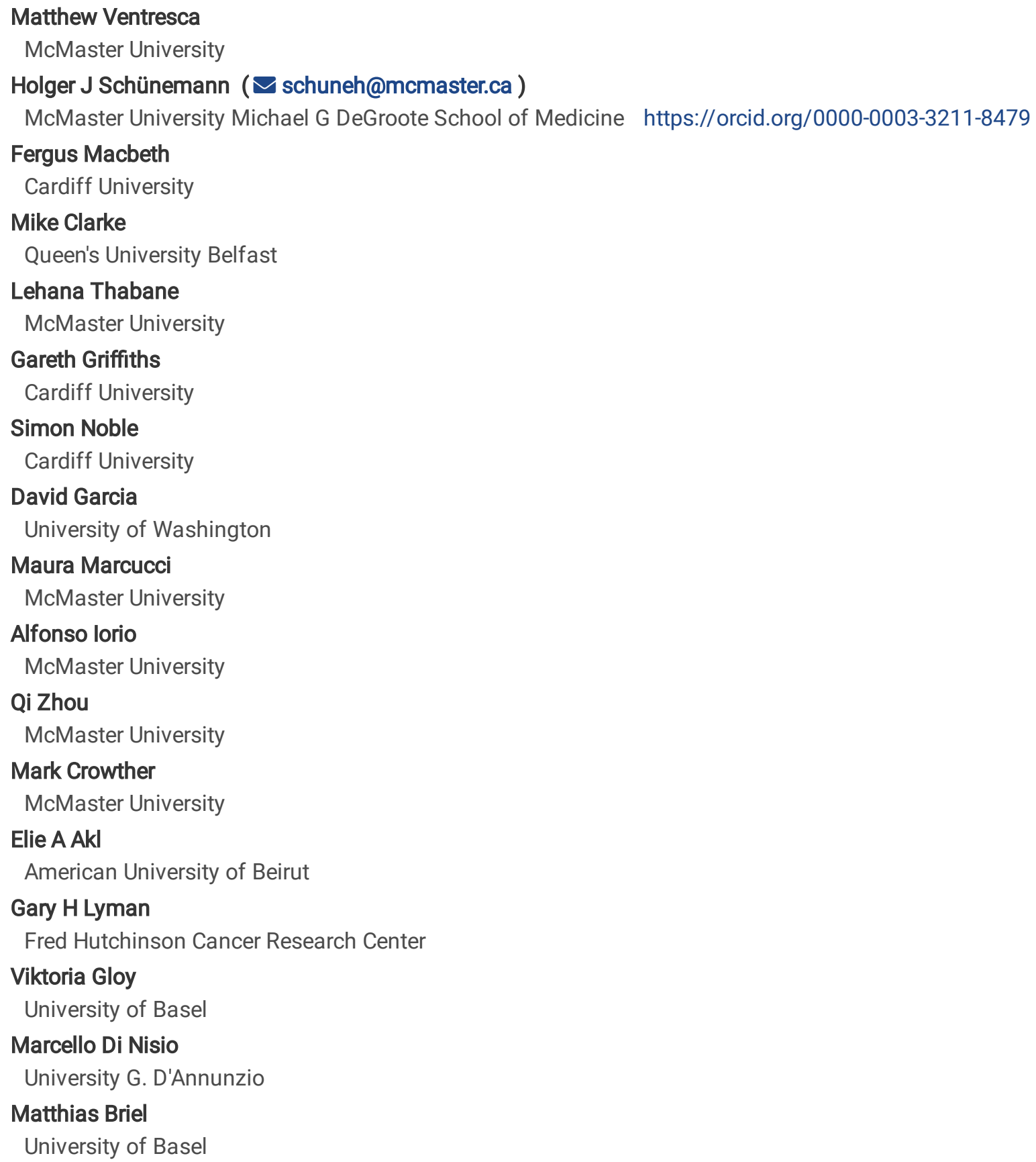


License: (c) (i) This work is licensed under a Creative Commons Attribution 4.0 International License. Read Full License

Version of Record: A version of this preprint was published at BMC Medical Research Methodology on May 12th, 2020. See the published version at https://doi.org/10.1186/s12874-020-00964-6. 


\section{Abstract}

Background Shifts in data sharing policy have increased researchers' access to individual participant data (IPD) from clinical studies. Simultaneously the number of IPD meta-analyses (IPDMAs) is increasing. However, rates of data retrieval have not improved. Our goal was to describe the challenges of retrieving IPD for an IPDMA and provide practical guidance based on a review of the literature and practical examples and observations.

Methods We systematically searched MEDLINE, Embase, and the Cochrane Library to identify publications focused on strategies to obtain IPD. In addition, we searched pharmaceutical websites and contacted industry organizations for supplemental information pertaining to recent advances in industry policy and practice. Finally, we documented setbacks and solutions encountered while completing a comprehensive IPDMA and drew on previous experiences related to seeking and using IPD.

Results Our scoping review identified 16 articles directly relevant for the conduct of IPDMAs. We present short descriptions of these articles alongside overviews of IPD sharing policies and procedures of pharmaceutical companies which display certification of Principles for Responsible Clinical Trial Data Sharing via Pharmaceutical Research and Manufacturers of America or European Federation of Pharmaceutical Industries and Associations websites. Advances in data sharing policy and practice affected the way in which data is requested, obtained, stored and analyzed For our IPDMA it took 6.5 years to collect and analyze relevant IPD and navigate additional administrative barriers. Delays in obtaining data were largely due to challenges in communication with study sponsors, frequent changes in data sharing policies of study sponsors, and the requirement for a diverse skillset related to research, administrative, statistical and legal issues.

Conclusions Knowledge of current data sharing practices and platforms as well as anticipation of necessary tasks and potential obstacles may reduce time and resources required for an IPDMA. Sufficient project funding and timeline flexibility are pre-requisites for successful collection and analysis of IPD. IPDMA researchers must acknowledge the additional and unexpected responsibility they are placing on study authors or data sharing administrators and should offer assistance in readying data for sharing.

\section{Introduction}

A meta-analysis aims to combine findings from different studies to obtain a more precise estimate of the average effect of an intervention or the size of an association, or to explore how and why results differ across studies [1]. There are several ways of synthesizing study data [2, 3], but individual participant data (IPD) meta-analyses (MAs), which synthesize IPD from multiple studies into a single dataset, are considered the "gold standard" [4-8]. IPDMA allows researchers to use the most current and comprehensive data, verify the findings of previous investigations, apply uniform definitions and analyses across studies, and avoid potential ecological bias when investigating interactions between interventions and patient-level characteristics (effect modifications, subgroup effects) [6, 7, 9-11]. IPDMAs often influence practice guidelines and the design of new trials [12, 13].

Ideally, an IPDMA should be based on IPD from all studies included in a systematic review [14]. However, fewer than half of systematic reviews with IPDMA, published between 1987 and 2015, retrieved data from at least $80 \%$ of relevant studies and from at least $80 \%$ of relevant participants [15]. The number of IPDMAs increased over this period [16], but data retrieval rates remained unchanged $[17,18]$. Failure to include eligible studies deviates from the purpose of a systematic review, decreases study power, and leads to healthcare decisions based on an incomplete, potentially biased data sample (studies with available data may differ from those whose data are not available) $[9,19,20]$. Since the first IPDMA guide published in 1995 [6], researchers have found that the process of obtaining, managing, and organizing IPD is typically the most resource intensive and time consuming step and may require years to complete $[1,6,17,21,22]$. Thus, many systematic reviews rely on aggregate level data even though sharing IPD and conducting IPDMA would be more useful [7, 20, 23-31].

Study participants have also understood the benefits of data sharing and are generally willing for this to happen, but may fear the loss of data confidentiality, misuse, or sharing without consent [32-35]. Governments [36, 37], research organizations [38-40], scientific journals $[38,41-46]$ and the pharmaceutical industry $[47,48]$ have developed data sharing policies. The Institute Of Medicine (IOM) has released four recommendations to guide responsible data sharing [49]: (1) maximize the benefits of clinical trials while minimizing the risks of sharing clinical trial data, (2) respect individual participants whose data are shared, (3) increase public trust in clinical trials and the sharing of trial data, and (4) conduct the sharing of clinical trial data in a fair manner.In July 
2013, amidst some criticism [50, 51], the European Federation of Pharmaceutical Industries and Associations (EFPIA) and the Pharmaceutical Research and Manufacturers of America (PhRMA) issued a joint statement describing the principles of responsible data sharing [47]. Several pharmaceutical companies and academic institutions are now working to handle data sharing requests in a more timely, better organized, and increasingly transparent manner [52-75].

Based on a review of the literature and our own experience with conducting IPDMAs, our goal was to provide practical guidance for researchers to successfully obtain IPD of eligible studies and to reduce resources required for IPDMA. We describe the key challenges and propose solutions to navigate obstacles commonly associated with IPDMA in the light of the latest policies $[15,76]$.

\section{Methods}

After delays during data acquisition for our recent IPDMA of the use heparin in patients with cancer, we noticed changes in policy and practice in clinical trial data access and began to log our setbacks and solutions [76]. We then conducted systematic searches of MEDLINE, Embase, and the Cochrane Library (from inception of each database until January 2019) to identify publications describing strategies to obtain IPD or IPDMA best practice. An experienced research librarian helped design a comprehensive search strategy using MeSH terms and text words (Appendix A) without any language restrictions.

We considered (1) articles describing IPDMA best practice including topics such as planning, cost, required time, common burdensome tasks, or administrative issues; (2) systematic reviews describing trends in IPDMA including topics such as IPD retrieval rates; (3) quantitative or qualitative studies describing strategies, barriers, or facilitators to obtain IPD from industry or investigator-sponsored studies; and (4) case reports describing authors' attempts to obtain IPD. We excluded IPDMAs reporting on a specific clinical question or statistical papers, e.g. studies describing different techniques of combining IPD with aggregate level data.

Two methodologically trained reviewers (MV and VG) independently screened titles and abstracts. If eligibility was suspected or unclear, we obtained full texts. Three reviewers (MV, MB, VG) screened full texts independently and in duplicate. Disagreements were resolved by discussion and consensus. From included articles we extracted information providing practical guidance for researchers to successfully obtain IPD and to make the conduct of IPDMA more efficient. Our scoping review adheres to the Preferred Reporting Items for Systematic reviews and Meta-Analyses extension for Scoping Reviews (PRISMA-ScR) guidelines [77].

Several publications examining specific data sharing issues outside of the context of an IPDMA (e.g. data sharing models or author reimbursement in general) did not meet the inclusion criteria for the scoping review but were referenced to provide additional context. In addition, we searched websites of pharmaceutical companies and industry organizations for press releases and other information about policies for sharing IPD. Finally, we drew on previous experiences related to providing, seeking, or using IPD. Based on the systematically identified literature, policy websites, and our own experience we developed practical guidance for IPDMA researchers that we structured according to the course of tasks when conducting an IPDMA.

\section{Results}

The systematic search of our scoping review yielded 3470 titles and abstracts (Figure 1).. We identified 16 eligible articles that are presented in Table 1 together with a short description. In the Box we summarize our main recommendations for researchers when retrieving data sets for IPDMAs and provide corresponding explanations and elaborations in the following sections.

\section{Box: Summary recommendations for obtaining individual participant data}

\section{Requesting data through personal contact or data sharing repository}

Review the data sharing policy of the study's sponsor organization.

Data sharing requests can be submitted via email or through a data sharing repository.

Contact data repositories to inquire about datasets not listed for request.

Page $4 / 30$ 
In addition to the IPD, consider requesting access for the study protocol, analysis plans, analysis-ready dataset, meta-data, annotated case report forms, and clinical study report.

Multiple contact attempts occurring over months or years may be required. Send emails on behalf of well-known researchers, those with personal connections to study authors, or from well-known research organizations to assist in garnering a response.

Discuss data sharing through teleconferences or in-person meetings rather than fragmented email correspondence whenever possible.

Offer to complete the essential data sharing tasks and provide necessary funding for researchers who may lack the time or organizational resources to share data.

Record the names, affiliations, contact information and roles of internal and external data sharing stakeholders throughout the data sharing process.

Incentives for data contributors

Offer authorship of the report of the IPDMA or other incentives to researchers who share data.

Setting up a data sharing agreement

Adapt previous data sharing agreements or existing templates to suit specific studies and institutional policies of study sponsors. Seek assistance form your institution's industry liaison office.

Time to data retrieval and refused requests

Continue to contact study stakeholders until a refusal to share data has been confirmed.

Seek reasoning for denied data sharing requests and attempt to develop solutions to data sharing barriers.

Effective communication and negotiation with primary study stakeholders may allow sharing of IPD before or immediately after publication of primary study results.

\section{Managing retrieved IPD}

Review the primary study protocol, results publications, clinical study reports, annotated case report forms and other shared files before and alongside data processing.

Datasets which could not be shared may be incorporated into analysis using methods which combine aggregate and IPD.

Allow data sharing organizations to review and comment on analysis prior to publication, ensuring accurate interpretation of shared data.

Identify projects emerging from IPDMA before results publication or prior to deletion of shared study data.

Confidentiality and data storage

Research local laws and sponsor policies pertaining to the storage and sharing of personally identifying information.

\section{Identifying relevant studies}

A sensitive search for all eligible studies, published and unpublished, is crucial for all systematic reviews to minimize publication bias [78]. Cochrane provides useful techniques to identify and obtain published as well as unpublished study data [14, 79]. Trial registries or regulatory bodies may be instrumental in identifying unpublished eligible studies and constitute an initial contact point (e.g. corresponding author or data sharing administrator) for data sharing requests. See Appendix $B$ for detailed information about the International Clinical Trials Registry Platform and Appendix $C$ on the United States Food and Drug Administration and the 
European Medicines Association. In principle, there are two approaches to obtain IPD: (1) direct contact with study authors, or (2) requests via a data repository [80].

The data collection process of our own IPDMA occurred between October 2012 and June 2016 [76].All data requests were placed by contacting study authors except for two of the 19 studies, which required use of the online data request portal clinicalstudydatarequest.com (CSDR). For all studies, we requested access to the clinical trial data, meta-data, study protocol, annotated case report forms, and clinical study report.

\section{Requesting study data through personal contact}

Analysis of data sharing requests submitted solely through study authors indicates that $58 \%$ of requests are successful [81]. Qualitative research examining useful techniques to obtain unpublished data indicates that polite requests which minimize additional responsibilities for the primary study author would be more likely to receive a response [82]. IPDMA authors typically attempt contact several times before quitting; the most persistent tried every 6 months for two to three years [80, 82-84]. From our own experience, obtaining data sets through personal contact may take between approximately four months and four years. Every corresponding author or study sponsor responded to our request; but we made repeated contact attempts via email, fax or phone. A description of our approach to correspondence and a sample email request are available in Table 2 and Appendix D, respectively.Email correspondence is often fragmented and delayed. We conducted phone or in-person meetings, e.g. at conferences, whenever more thorough discussions of specific issues were necessary. Authors may lack time or organizational resources and this requires funding to offer support with essential data sharing tasks (e.g. transferring data to an electronic format, drafting data sharing agreement). Recording contact information and roles of data sharing stakeholders (e.g. administrators, statisticians, industry liaisons, ethical and legal representatives) is essential. This will ease subsequent communication which often occurred years after the first data request as the IPDMA progressed to publication. In some cases, we reviewed the institution's data sharing request policy to identify additional study stakeholders or alternative request procedures.

\section{Requesting study data via data repository or data sharing administrator}

In our IPDMA, two datasets were requested and approved through CSDR, a consortium of clinical study sponsors and funders which facilitates responsible data sharing [85]. Initially, neither dataset was available on request, but this did not preclude the data from being shared. Determining whether a study can be shared may require IPDMA authors to directly contact CSDR administrators and submit a full study proposal, as we did, rather than making a simple inquiry or scanning a registry [86]. In one case, data were not available because the sponsor had not yet properly curated the data but our request hastened this process. In the second, study sponsors were in the process of establishing a presence on CSDR.

The process of submitting data requests on CSDR takes approximately 30 to 60 minutes; it was intuitive, and directions were available $[87,88]$. Our request package identified the specific study by the title and National Clinical Trial number and included our study protocol, timeline, funding sources, description of research team members' experience and roles, conflicts of interest, and publication plans. Knowledge of jurisdictional laws (e.g. Personal Information Protection and Electronic Documents Act and General Data Protection Regulation) and collaboration with legal representatives was required before submitting data sharing requests and while negotiating data sharing agreements. Approximately 4 months were needed to process each data sharing request and finalize the data sharing agreement, consistent with CSDR estimates [89, 90]. After finalizing the data sharing agreement, our questions pertaining to data sharing processes or system technical difficulties were typically responded to within one day.

As of December 31, 2018, 1161 requests were made for data not readily advertised on CSDR; 476 submissions were approved and 640 denied, while 17 are still under consideration [91]. Of companies which have received at least 40 requests for non-listed studies, the reported lowest percentage of approval is 9\%, (Eisai), and the highest $80 \%$ (GlaxoSmithKline) [91]. Geifman et al. reported the data request process via CSDR to be unnecessarily lengthy, while requests submitted through Project Data Sphere required only days before data access was provided [92]. The joint PhRMA and EFPIA statement represents the minimum clinical transparency standard, but participation is voluntary [47] [93] [94]. Industry sponsors which are members of PhRMA or EFPIA are more likely to publicize a data sharing policy and make trial data eligible for sharing $[95,96]$. 
Data access points, summary of data made available, and date from which the pharmaceutical company's IPD sharing policy applies are exhibited in Table 3 and Table 4. Each sponsor's specific policy should be referred to for a complete review of available data. A sponsor's exclusion from Table 3 or Table 4 is not meant to indicate they are not wholly committed to data sharing, but that as of March 5, 2019, certification of their compliance with the Principles for Responsible Clinical Trial Data Sharing was not confirmed through PhRMA or EFPIA websites $[98,99]$. Repositories may also provide access to study data which is sponsored, generated or stored by governments, universities, charities and research organizations $[52,100]$.

Of certified pharmaceutical companies, 23 use at least one internal or external online portal to manage data sharing requests, including clinicalstudydatarequest.com (15), vivli.org (8), yoda.yale.edu (1),fasttrack-bms.force.com (1), https://biogen-dtexternal.pharmacm.com/DT/Home (1) and purduepharma.com/healthcare-professionals/clinical-trials/request-trial-data (1). Data requests for the remainder of certified pharmaceutical companies are solicited via email. In Table 5 we describe the data request review processes from each pharmaceutical company certified through PhRMA or EFPIA. As of March 31, 2019, 3682 studies were available on request through CSDR [91]. The recently launched data sharing platform Vivli lists over 3100 studies [101].

\section{Incentives for data contributors}

Study authors and data curators who generated, managed and shared data, and provided commentary on findings make considerable efforts that should be recognized. Given the role in data collection and interpretation of data, we offered primary investigators authorship on relevant publications. Indeed, researchers generally agree that trialists who share data deserve recognition and propose several recognition models, some including penalties for those who refuse data sharing [27, 82, 102-109]. Authorship also enables primary researchers to contribute to the manuscript before publication and reduces anxiety about a lack of control over data and fellow researchers' ability to understand shared data or IPDMA results $[106,107]$.

There are several administrative, standardization, human resources and opportunity costs to properly preserve a data repository, manage requests and prepare data for additional analysis which IPDMA authors may be asked to contribute to [110-114]. Academic researchers are expected to pay between $\$ 30,000$ and $\$ 50,000$ annually to list up to 20 studies on CSDR [115]. Vivli asks researchers and pharmaceutical companies to pay between $\$ 2,000$ and $\$ 4,500$ per listed study [116]. We obtained funding to offer reimbursement of costs associated with data sharing (e.g. shipping fees) but did not offer direct payment solely for sharing data which was also not requested by any of the collaborating parties. Offering small financial incentives to primary study authors has not improved IPD retrieval rates [84].

\section{Setting up a data sharing agreement}

Data sharing agreements describe the conditions which the IPDMA research team should respect in exchange for permission to analyze specified data from a trialist or study sponsor, and are recommended when sharing data $[49,117,118]$. Data sharing agreements include the study rationale, analysis plan, contents being exchanged, participant confidentiality, timing of data sharing, data storage and security measures, third party data sharing, intellectual property rights, publication plans and authorship, among others. We adapted previous data sharing agreements to suit the institutional policies of respective study sponsors. We sought feedback from our institution's industry liaison department regarding legal phrasing and implications of the data sharing agreement. Appendix E presentsan example data sharing agreement with further details. We had to negotiate amendments to ratified agreements if institutional policies changed, if there were data sharing issues affecting agreements with others, or when we conducted additional analyses.

\section{Time to data retrieval and reasons for refused requests}

Two of our data sharing requests were not granted (one because of ongoing analyses and the other because it could not be transferred to an electronic format) and three could not be pursued because of timeline and resource restriction. This meant that we were unable to obtain data for $18 \%$ ofparticipants $(n=1,763)$ [76]. Contacting trial authors, negotiating data sharing agreements and awaiting publication of study results are common reasons for delays. Approximately $43 \%$ of IPDMAs obtain at least $80 \%$ of IPD

[15]. The IOM recommends that sponsors make available the "full data package" to external researchers no later than 18 months 
after trial completion and the "post-publication data package" no more than 6 months after trial completion [49]. In practice, the time until IPD become available after trial completion varies greatly. This availability is influenced by when primary results are published, a drug's development program is terminated or approved by regulators, among other factors [52].

Data which are commonly unavailable on request to pharmaceutical companies include commercially confidential information, and study data which were not submitted as part of a marketing authorization package [52]. Sponsors may require that secondary analysis investigate the same indication as primary analysis because study participants have not provided consent for other investigations. Many sponsors have recognized this impediment and changed their participant consent forms accordingly [52]. Systematic reviews have identified several other technical, motivational, economic, political, legal and ethical barriers to data sharing $[15,113,119,120]$.

Authors' motivations for accepting or rejecting data sharing requests include advancing science, improving health, complying with employer, funding, sponsor policies, perceived effort and personal recognition [20, 25, 49, 106, 107, 119-125]. Some argued that older trials require excessive time and resources to properly anonymize IPD, update databases to current standard or transfer data to an electronic format, assuming they have not been lost $[15,84]$. Sharing of databases may be refused because datasets are too large to properly anonymize and transfer to other researchers $[52,126]$.

If a request is denied, IPDMA researchers may combine IPD with aggregate data to examine the potential impact of studies without IPD on results and understand the totality of the evidence [3, 19, 127-129].

\section{Managing retrieved IPD}

Reviewing supplemental material and readying datasets is a time consuming and resource intensive task [112]. Older datasets generally require additional maintenance as they are not digitally recorded or coded to current standards. For our IPDMA, we reviewed the study protocol, publications, clinical study reports, annotated case report forms and other shared files, before and alongside data extraction to understand the dataset and ensure accuracy. Annotated case report forms are particularly helpful in understanding shared data as they connect each specific variable in a dataset to when, why, where, or how the data was collected when data files contain large numbers of variables. We logged inconsistencies and typically resolved them through discussion with study stakeholders (e.g. trial coordinators). Important inconsistencies should be described in publications following the Preferred Reporting Items for Systematic Review and Meta-Analyses of Individual Participant Data (PRISMA-IPD) statement [130].

We created a unified database that was verified by two researchers. Our data sharing agreements require that shared data will be deleted within six months of results publication which requires careful planning of all analyses.

Access to one study required use of the SAS Clinical Trial Data Transparency (CTDT) portal and approval from the institutional review board and trial sponsors [131, 132]. A manual is provided to assist researchers using the CTDT portal, but training is needed if researchers are unfamiliar with statistical analysis programs [132-135]. A dedicated support team is available to resolve technical issues. The consent of sponsors not using the SAS CTDT system may be required before uploading data to this interface. Conversely, IPDMA researchers may also try to negotiate the download of data typically securely accessed through the CTDT system. For further review of methodology and statistical issues for IPDMA see Debray et al. 2015 [128].

\section{Confidentiality and data storage}

In our IPDMA, we deleted information from databases that identified study participants (e.g. names or phone numbers) because storing personal information is not in the interest of study participants. Indeed, the general public and study participants worry about storing or sharing of personally identifying information, obtaining appropriate consent to use data, and relationships with the study investigators $[26,34,136]$. IPDMA researchers must be aware of local laws and sponsor policies about the storage of personally identifying information [15]. Concerns about lack of anonymity are also common when requesting data from casestudies or case-series involving fewer than 50 participants, trials of rare diseases or trials assessing genomic data [52]. Thus, all data requires storage on secure password protected servers where access is provided only to those directly involved in data analysis according to available standards [52, 137-139].

Page $8 / 30$ 


\section{Discussion}

We conducted a scoping review of challenges and solutions to obtaining and using IPD and supplemented this by descriptions of our own experiences to guide and facilitate future IPDMA. Many of the practical issues are new compared to the Cochrane IPDMA working group's guide published by Stewart and Clarke in 1995 [6]. Technological and cultural changes have modified the ways in which researchers communicate and collaborate and the ways data are shared, managed and analyzed. Recent guidance on the use and appraisal of IPDMAs [140, 141], reporting standards [130], data sharing [49], and statistical techniques [128] have influenced these policies.

Our IPDMA identified 19 eligible studies and 10,032 eligible participants which is above the median of typical IPDMAs (i.e. 14 eligible studies and 2,369 participants) [15]. Unexpected delays throughout the data gathering process resulted from challenges in communication and the need to adapt to modifications in the various sponsors' data sharing practices, which were evolving alongside industry and government policy. These changes included the joint PhRMA/EFPIA statement on the principles of responsible clinical trial data sharing [47], launch of the AllTrials campaign [142], GlaxoSmithKline introducing the first online data request platform before transitioning to CSDR in 2014 [143], and influential publications highlighting the importance of data sharing and open science [144-146].

\section{Limitations}

One limitation is that this manuscript was not planned before starting the IPDMA we use as primary example in this work but because of the many challenges, we were encouraged to provide guidance. Thus, our solutions are based on firsthand experiences but have not been formally compared to alternatives and may not be applicable to all IPDMA. Our perspective is that of IPDMA researchers and not of trialists, sponsors, or data sharing administrators who may disagree with our proposals. Other IPDMA or study stakeholders may identify additional obstacles or solutions not described here but we have conducted a scoping review to overcome that limitation.

\section{Relation To Other Studies}

We identified several publications which aimed to provide a firsthand description of specific data sharing experiences $[15,23,92$, 147-149]. For example, Savage and Vickers obtained only one of 10 requested studies and established contact with only five of 10 corresponding authors [148]. Data from four studies were not shared because preparation was too laborious, data were forbidden from being shared, or required an extensive proposal submission [148]. Jaspers and Degraeuwe described their attempt to conduct an IPDMA, which was eventually abandoned because they were able to obtain only 40\% of IPD. Barriers to accessing data were similar to those we describe here and included difficulties establishing contact with study authors, denial of requests for raw datasets because of ongoing analysis or because of a lack of time and personnel to properly prepare data. Geifman et al. and Filippon et al. reported costly and repeated data sharing requests [92, 149]. Nevitt et al. performed a systematic review of IPDMAs published between 1987 and 2015, and reported that only 25\% of published IPDMAs had access to all identified IPD and no improvement in data retrieval rate over time [15]. IPDMAs were associated with retrieving at least $80 \%$ of IPD if they included only randomized trials, had an authorship policy which provided an incentive to share data (e.g. co-authorship), included fewer eligible participants, and were not Cochrane Reviews.

\section{Conclusions}

As shifts in data sharing policy and practice continue, IPDMA researchers must be prepared to mitigate the effects of project delays. Knowledge of how to establish and maintain contact with study stakeholders, negotiate data sharing agreements, and manage clinical study data is required. Broader issues including designing trials for secondary analysis, participant confidentiality, data sharing models, data sharing platforms, data request review panels and recognition of primary study investigators must also be understood to ensure an IPDMA is conducted to appropriate scientific, ethical, and legal standard [150-159]. We hope that a shift away from peer-to-peer requesting procedures towards data repository requests will help [160]. The discussion of specific data sharing issues such as the effectiveness of data sharing policies [161], output of data sharing endeavours [162], confidentiality of commercial information, whom data is shared with, timelines for data requests, and appropriately compensating data sharing parties must continue [26, 27, 153, 163-166]. Additional research investigating the effectiveness of data acquisition techniques 
[167], platform features which aid the sharing of clinical trial data [168-170], incentives for data sharing [123, 161], participant broad consent and data sharing [171] is needed.

\author{
Abbreviations \\ CSDR-Clinicalstudydatarequest.com \\ CTDT-Clinical trial data transparency \\ EFPIA - European Federation of Pharmaceutical Industries and Associations \\ Embase - Excerpta Medica dataBASE \\ IOM-Institutes of Medicine \\ IPDMA-Individual participant data meta-analysis \\ MEDLINE-Medline Literature Analysis and Retrieval System Online \\ PhRMA - Pharmaceutical Research and Manufacturers of America \\ PRISMA-IPD-Preferred Reporting Items for a Systematic Review and Meta-Analysis of Individual Participant Data \\ SAS-Statistical Analysis System \\ US-United States
}

\title{
Declarations
}

\section{Ethics approval and consent to participate}

Not applicable

\section{Consent for publication}

Not applicable

\section{Availability of data and material}

All data generated or analysed during this study are included in this published article and its supplementary information files.

\section{Competing interests}

Dr. Clarke reports being co-convenor of the Cochrane Individual Participant Data Meta-analysis Methods Group and involved in several IPDMAs funded by a variety of research funders. Dr. Garcia reports grants and personal fees from Incyte, grants from Bayer, grants, personal fees and non-financial support from Janssen, personal fees and non-financial support from Seattle Genetics, grants from Daiichi Sankyo, outside the submitted work. Dr. Di Nisio reports personal fees from Bayer, personal fees from BMSPfizer, personal fees from Leo Pharma, personal fees from Aspen, personal fees from Daiichi Sankyo, outside the submitted work. Dr. Noble reports grants from Leo Pharma, personal fees from Bayer, personal fees from Daiichi Sankyo, outside the submitted work. The remaining authors declare that they have no competing interests.

\section{Funding}


Funding for this project was supplied through a grant from the Canadian Institutes of Health Research (CIHR), KRS 126594. The funding body had no role in the design of the study and collection, analysis, and interpretation of data and in writing the manuscript.

\section{Authors' contributions}

MV contributed to study conception and gathering of relevant information, and wrote the first draft of the manuscript. HJS conceived of the study, contributed to gathering of relevant information, supervision of the study, and writing of the manuscript. MB contributed to study conception, supervision of the study, and writing of the manuscript. All authors read, provided feedback, and approved the final manuscript.

\section{Acknowledgements}

We thank Neera Bhatnagar (Health Sciences Library, McMaster University) for her help with designing the search strategy for our scoping review.

\section{References}

1.Clarke, M. J., Individual patient data meta-analyses. Best Pract Res Clin Obstet Gynaecol, 2005. 19(1): p. 47-55.

2.Cooper, H. M., L. V. Hedges, and J. C. Valentine, The handbook of research synthesis and meta-analysis. 2nd ed. 2009, New York: Russell Sage Foundation. xvi, 615 p.

3.Riley, R. D., M. C. Simmonds, and M. P. Look, Evidence synthesis combining individual patient data and aggregate data: a systematic review identified current practice and possible methods. J Clin Epidemiol, 2007. 60(5): p. 431-9.

4.Simmonds, M. C., et al., Meta-analysis of individual patient data from randomized trials: a review of methods used in practice. Clin Trials, 2005. 2(3): p. 209-17.

5.Tudur Smith, C., et al. Individual participant data meta-analyses compared with meta-analyses based on aggregate data [abstract]. Trials, 2011. 12, 21-2.

6.Stewart, L. A. and M. J. Clarke, Practical methodology of meta-analyses (overviews) using updated individual patient data. Cochrane Working Group. Stat Med, 1995. 14(19): p. 2057-79.

7.Stewart, L. A. and J. F. Tierney, To IPD or not to IPD? Advantages and disadvantages of systematic reviews using individual patient data. Eval Health Prof, 2002. 25(1): p. 76-97.

8.Tudur Smith, C., et al., Individual participant data meta-analyses compared with meta-analyses based on aggregate data. Cochrane Database Syst Rev, 2016. 9: p. MR000007.

9.Lyman, G. H. and N. M. Kuderer, The strengths and limitations of meta-analyses based on aggregate data. BMC Med Res Methodol, 2005. 5: p. 14.

10.Mello, M. M., et al., Preparing for responsible sharing of clinical trial data. N Engl J Med, 2013. 369(17): p. 1651-8.

11.Stewart LA, T. J., Clarke M. Chapter 18: Reviews of individual patient data. In: Higgins JPT, Green S (editors), Cochrane Handbook for Systematic Reviews of Interventions Version 5.1.0 (updated March 2011). The Cochrane Collaboration, 2011; Available from: www.handbook.cochrane.org.

12.Vale, C. L., et al., Uptake of systematic reviews and meta-analyses based on individual participant data in clinical practice guidelines: descriptive study. BMJ, 2015. 350: p. h1088. 
13.Tierney, J. F., et al., How individual participant data meta-analyses have influenced trial design, conduct, and analysis. J Clin Epidemiol, 2015. 68(11): p. 1325-35.

14.Higgins JPT and Green S. Cochrane Handbook for Systematic Reviews of Interventions Version 5.1.0 [updated March 2011]. [cited 2018 January 11]; Available from: www.handbook.cochrane.org.

15.Nevitt, S. J., et al., Exploring changes over time and characteristics associated with data retrieval across individual participant data meta-analyses: systematic review. BMJ, 2017. 357: p. j1390.

16.Coady, S. A., et al., Use of the National Heart, Lung, and Blood Institute Data Repository. New England Journal of Medicine, 2017. 376(19): p. 1849-1858.

17.Nevitt, S. J., et al., Exploring changes over time and characteristics associated with data retrieval across individual participant data meta-analyses: Systematic review. BMJ (Online), 2017. 357 (no pagination)(j1390).

18.Huang, Y., et al., Distribution and epidemiological characteristics of published individual patient data meta-analyses. PLoS One, 2014. 9(6): p. e100151.

19.Ahmed, I., A. J. Sutton, and R. D. Riley, Assessment of publication bias, selection bias, and unavailable data in meta-analyses using individual participant data: a database survey. BMJ, 2012. 344: p. d7762.

20.Rathi, V., et al., Sharing of clinical trial data among trialists: a cross sectional survey. BMJ, 2012. 345: p. e7570.

21.Riley, R. D., P. C. Lambert, and G. Abo-Zaid, Meta-analysis of individual participant data: rationale, conduct, and reporting. BMJ, 2010. 340: p. c221.

22.Abo-Zaid, G., W. Sauerbrei, and R. D. Riley, Individual participant data meta-analysis of prognostic factor studies: state of the art? BMC medical research methodology, 2012. 12: p. 56.

23.Jaspers, G. J. and P. L. Degraeuwe, A failed attempt to conduct an individual patient data meta-analysis. Syst Rev, 2014. 3: p. 97.

24.Tenopir, C., et al., Changes in Data Sharing and Data Reuse Practices and Perceptions among Scientists Worldwide. PLoS One, 2015. 10(8): p. e0134826.

25.Rathi, V. K., et al., Predictors of clinical trial data sharing: exploratory analysis of a cross-sectional survey. Trials, 2014. 15 : p. 384.

26.Ross, J. S., Clinical research data sharing: what an open science world means for researchers involved in evidence synthesis. Syst Rev, 2016. 5(1): p. 159.

27.Ohmann, C., et al., Sharing and reuse of individual participant data from clinical trials: principles and recommendations. BMJ Open, 2017. 7(12): p. e018647.

28.Barnhart, K. T., R. S. Legro, and R. T. Scott, Jr., Data sharing requirements: perspectives from three authors. Fertil Steril, 2018. 109(1): p. 44-47.

29.Sardanelli, F., et al., To share or not to share? Expected pros and cons of data sharing in radiological research. European Radiology, 2018. 28(6): p. 2328-2335.

30.Wu, T., et al., Transparency and sharing individual participant data of clinical trials: A philosophical proposition about the medical study ethics and implications for clinical trials. [Chinese]. Chinese Journal of Evidence-Based Medicine, 2018. 18(6): p. 538-542.

31.Vickers, A. J., Sharing raw data from clinical trials: what progress since we first asked "Whose data set is it anyway?". Trials [Electronic Resource], 2016. 17(1): p. 227. 
32.Bell, E. A., L. Ohno-Machado, and M. A. Grando, Sharing my health data: a survey of data sharing preferences of healthy individuals. AMIA Annu Symp Proc, 2014. 2014: p. 1699-708.

33.Cheah, P. Y., et al., Perceived Benefits, Harms, and Views About How to Share Data Responsibly: A Qualitative Study of Experiences With and Attitudes Toward Data Sharing Among Research Staff and Community Representatives in Thailand. J Empir Res Hum Res Ethics, 2015. 10(3): p. 278-89.

34. Howe, N., et al., Systematic review of participants' attitudes towards data sharing: A thematic synthesis. Journal of Health Services Research and Policy, 2018. 23(2): p. 123-133.

35.Mello, M. M., V. Lieou, and S. N. Goodman, Clinical trial participants' views of the risks and benefits of data sharing. New England Journal of Medicine, 2018. 378(23): p. 2202-2211.

36.European Medicines Agency. European Medicines Agency policy on publication of clinical data for medicinal products for human use. 2014 [cited 2014 October 3]; Available from:

http://www.ema.europa.eu/docs/en_GB/document_library/Other/2014/10/WC500174796.pdf.

37.Food and Drug Administration (FDA). Availability of Masked and De-identified Non-Summary Safety and Efficacy Data; Request for Comments. 2013 [cited 2014 June 20]; Available from: https://federalregister.gov/a/2013-13083.

38.Taichman, D. B., et al., Data Sharing Statements for Clinical Trials: A Requirement of the International Committee of Medical Journal Editors. PLoS Med, 2017. 14(6): p. e1002315.

39.Cancer Research UK. Submission of a data sharing and preservation strategy. 2009 June 1, 2015]; Available from:

http://www.cancerresearchuk.org/prod_consump/groups/cr_common/@fre/@gen/documents/generalcontent/cr_016308.pdf.

40.Kiley, R., et al., Data Sharing from Clinical Trials - A Research Funder's Perspective. New England Journal of Medicine, 2017. 377(20): p. 1990-1992.

41.International Committee of Medical Journal Editors. Clinical Trials. 2018 [cited 2018 July 1]; Available from: http://www.icmje.org/recommendations/browse/publishing-and-editorial-issues/clinical-trial-registration.html.

42.Godlee, F. and T. Groves, The new BMJ policy on sharing data from drug and device trials. BMJ, 2012. 345: p. e7888.

43.PLOS Medicine. PLOS editorial and publishing policies. [cited 2014 July 1]; Available from: http://www.plosmedicine.org/static/policies.action;jsessionid = OCD27C4DE9841485620ED21B9E815D54\#sharing.

44.Scientific Data. Welcome to Scientific Data. [cited 2014 July 1]; Available from: http://www.nature.com/sdata/about.

45.Loder, E. and T. Groves, The BMJ requires data sharing on request for all trials. BMJ (Clinical research ed.), 2015. 350: p. h2373.

46.Fletcher, J., New CMAJ policy on sharing data from clinical research. Cmaj, 2014. 186(3): p. 163.

47.European Federation of Pharmaceutical Industries and Associations (EFPIA) and Pharmaceutical Research and Manufacturers of America (PhRMA). Principles for resposible clinical trial data sharing: Our commitment to patients and researchers. 2013 [cited 2018 July 30]; Available from: http://phrma-

docs.phrma.org/sites/default/files/pdf/PhRMAPrinciplesForResponsibleClinicalTrialDataSharing.pdf.

48.Atzor, S., et al., Clinical trial data sharing: From principles to practical implementation - An industry model. Regulatory Rapporteur, 2014. 11(4): p. 4-7.

49.Lo, B., Sharing clinical trial data: maximizing benefits, minimizing risk. JAMA, 2015. 313(8): p. 793-4.

50.0'Dowd, A., Drug industry pledge on access to trial data is met with scepticism. BMJ, 2013. 347: p. f4829.

51.Doshi, P., EFPIA-PhRMA's principles for clinical trial data sharing have been misunderstood. BMJ, 2013. 347: p. f5164.

Page 13/30 
52.Clinical Study Data Request. Study sponsors. [cited 2014 July 4]; Available from: https://www.clinicalstudydatarequest.com/Study-Sponsors.aspx.

53.Abbvie. Clinical trials data and information sharing. 2014 [cited 2019 March 21]; Available from: https://www.abbvie.com/ourscience/clinical-trials/clinical-trials-data-and-information-sharing/data-and-information-sharing-with-qualified-researchers.html.

54.Amgen. Clinical Trial Transparency, Data Sharing and Disclosure Practices 2019 [cited 2019 March 14]; Available from: https://www.amgen.com/about/how-we-operate/policies-practices-and-disclosures/ethical-research/clinical-data-transparencypractices/.

55.Astellas. About Astellas Clinical Study Results. 2018 [cited 2019 March 1]; Available from: https://astellasclinicalstudyresults.com/HCP.aspx.

56.Bayer. Clinical trial transparency policy. [cited 2019 March 12]; Available from: http://pharma.bayer.com/en/innovationpartnering/clinical-trials/transparency-policy/.

57.Biogen. Biogen clinical trial transparency and data sharing policy. 2018 [cited 2018 July 30]; Available from: http://clinicalresearch.biogen.com/.

58.Boehringer-Ingelheim. Policy on transparency and publication of clinical study data: Registration of clinical studies, disclosure of results in publications and results databases and access to study data and documents. [cited 2019 March 12]; Available from: https://trials.boehringer-ingelheim.com/content/dam/internet/opu/clinicaltrial/com_EN/documents/Policy.pdf.

59.Bristol-Myers Squibb. Disclosure Commitment. 2018 [cited 2018 July 30]; Available from: https://www.bms.com/researchersand-partners/clinical-trials-and-research/disclosure-commitment.html.

60.Celgene. Clinical trials data sharing. 2019 [cited 2019 March 14]; Available from: https://www.celgene.com/researchdevelopment/clinical-trials/clinical-trials-data-sharing/.

61.Eisai Co. Ltd. Clinical trial disclosure. [cited 2019 March 12]; Available from: https://www.eisai.com/company/business/research/clinical/index.html.

62.Eli Lilly and Company. Lilly announces increased access to clinical trials data for qualified researchers. 2014 [cited 2019 March 12]; Available from: http://lilly.mediaroom.com/index.php?s = 9042\&item = 137313.

63.EMD Serono. Our Commitment to Responsible Clinical Trial Data Sharing. 2018 [cited 2018 July 30]; Available from: https://www.emdgroup.com/en/research/our-approach-to-research-and-development/healthcare/clinical-trials/commitmentresponsible-data-sharing.html.

64.GlaxoSmithKline. GSK gives update on plans to share detailed clinical trial data as part of its commitment to transparency. 2013 [cited 2019 March 12]; Available from: https://www.gsk.com/en-gb/media/press-releases/gsk-gives-update-on-plans-to-sharedetailed-clinical-trial-data-as-part-of-its-commitment-to-transparency/.

65.Viiv Healthcare. We are committed to data transparency. 2019 [cited 2019 March 13]; Available from:

https://www.viivhealthcare.com/en-gb/advancing-hiv-science-and-rd/we-are-committed-to-data-transparency/.

66.Pfizer. Data access requests. 2019 [cited 2019 March 1]; Available from:

https://www.pfizer.com/science/clinical_trials/trial_data_and_results/data_requests.

67.Sanofi. Clinical trials and results: Our data sharing commitments. 2019 [cited 2019 March 13]; Available from: https://www.sanofi.com/en/science-and-innovation/clinical-trials-and-results/our-data-sharing-commitments/.

68.Shire. Our commitment to transparency. 2017 [cited 2019 March 14]; Available from: http://www.shiretrials.com/en/ourcommitment-to-transparency/data-sharing-with-researchers. 
69.Takeda. A Tradition of Serving Patients With Integrity. 2015 [cited 2019 March 13]; Available from: https://takedaclinicaltrials.com/approach.

70.UCB. UCB position statement on clinical study data transparency. 2014 [cited 2014 July 1]; Available from:

https://www.ucb.com/our-science/magazine/detail/article/Transparency-supporting-responsible-data-sharing.

71.The Yale University Open Data Access Project. Johnson \& Johnson - Available Data. 2018 [cited 2018 July 30]; Available from: http://yoda.yale.edu/jj-available-data.

72.Janssen Global Services, L. Clinical trial data transparency. 2018 [cited 2018 July 30]; Available from:

https://www.janssen.com/clinical-trials/transparency.

73.Merck. Procedure on access to clinical trial data. 2018 [cited 2019 March 22]; Available from: https://www.merck.com/clinicaltrials/pdf/ProcedureAccessClinicalTrialData.pdf.

74.Purdue Pharma. Review process for data sharing. 2018 [cited 2019 March 21]; Available from:

http://purduepharma.com/healthcare-professionals/clinical-trials/data-sharing/.

75. Roche. Roche global policy on sharing of clincal study information. [cited 2019 March 13]; Available from:

https://www.roche.com/dam/jcr:1c46aa73-cea0-4b9b-8eaa-

e9a788ed021b/en/roche_global_policy_on_sharing_of_clinical_study_information.pdf.

76.Schunemann, H. J., et al., Use of heparins in patients with cancer: individual participant data meta-analysis of randomised trials study protocol. BMJ Open, 2016. 6(4): p. e010569.

77.Tricco, A. C., et al., PRISMA Extension for Scoping Reviews (PRISMA-SCR): Checklist and Explanation. Ann Intern Med, 2018. 169(7): p. 467-473.

78.Lefebvre C, M. E., Glanville J. Chapter 6: Searching for studies In: Higgins JPT, Green S (editors). Cochrane Handbook for Systematic Reviews of Interventions Version 5.1.0 (updated March 2011). The Cochrane Collaboration, 2011; Available from: www.handbook.cochrane.org.

79.Young, T. and S. Hopewell, Methods for obtaining unpublished data. Cochrane Database Syst Rev, 2011(11): p. MR000027.

80.Polanin, J. R. and R. T. Williams, Overcoming obstacles in obtaining individual participant data for meta-analysis. Research synthesis methods, 2016. 7(3): p. 333-341.

81.Polanin, J. R., Efforts to retrieve individual participant data sets for use in a meta-analysis result in moderate data sharing but many data sets remain missing. Journal of Clinical Epidemiology, 2018. 98: p. 157-159.

82.Wolfe, N., P. C. Gotzsche, and L. Bero, Strategies for obtaining unpublished drug trial data: a qualitative interview study. Syst Rev, 2013. 2: p. 31.

83.Polanin, J. R. and M. Terzian, A data-sharing agreement helps to increase researchers' willingness to share primary data: results from a randomized controlled trial. Journal of Clinical Epidemiology, 2019. 106: p. 60-69.

84.Veroniki, A. A., et al., Retrieval of individual patient data depended on study characteristics: a randomized controlled trial. J Clin Epidemiol, 2019. 113: p. 176-188.

85.Clinical Study Data Request. Our mission. 2019 [cited 2019 April 1]; Available from:

https://clinicalstudydatarequest.com/Default.aspx.

86.Murugiah, K., et al., Availability of Clinical Trial Data From Industry-Sponsored Cardiovascular Trials. Journal of the American Heart Association, 2016. 5(4): p. e003307.

Page $15 / 30$ 
87.Clinical Study Data Request. How to request data. 2018 [cited 2018 July 30]; Available from:

https://www.clinicalstudydatarequest.com/Help/Help-How-to-Request-Data.aspx.

88.NHS Digital. DARS guidance. 2019 [cited 2019 February 22]; Available from: https://digital.nhs.uk/services/data-access-requestservice-dars/dars-guidance.

89.Clinical Study Data Request. Review of requests. 2018 [cited 2018 July 30]; Available from:

https://www.clinicalstudydatarequest.com/Help/Help-Review-of-Requests.aspx.

90.NHS Digital. Data Access Request Service (DARS): process. 2019 [cited 2019 February 22]; Available from:

https://digital.nhs.uk/services/data-access-request-service-dars/data-access-request-service-dars-process.

91.Clinical Study Data Request. Metrics. 2019 [cited 2019 April 1]; Available from:

https://clinicalstudydatarequest.com/Metrics.aspx.

92.Geifman, N., et al., Opening clinical trial data: Are the voluntary data-sharing portals enough? BMC Medicine, 2015. 13 (1) (no pagination)(280).

93.Pharmaceutical Research and Manufacturers of America (PhRMA). Members. 2019 [cited 2019 March 4]; Available from: https://www.phrma.org/about/members.

94.European Federation of Pharmaceutical Industries and Associations (EFPIA). Membership. 2019 [cited 2019 March 4]; Available from: https://www.efpia.eu/about-us/membership/.

95.Hopkins, A.M., A. Rowland, and M. J. Sorich, Data sharing from pharmaceutical industry sponsored clinical studies: Audit of data availability. BMC Medicine, 2018. 16 (1) (no pagination)(1154).

96.Pharmaceutical Research and Manufacturers of America (PhRMA). EFPIA-PHRMA Princples for responsible clinical trial data sharing report on the 2016 member company survey. 2017 [cited 2019 March 4]; Available from:

https://www.efpia.eu/media/288603/efpia-phrma-report-on-the-2016-member-company-survey-on-the-joint-principles-forresponsible-clinical-trial-data-sharing.pdf.

97.European Federation of Pharmaceutical Industries and Associations (EFPIA). EFPIA Annual Report 2017. 2018 [cited 2019 March 4]; Available from: https://www.efpia.eu/media/362132/efpia_annual-report_2018_interactive-1.pdf.

98.European Federation of Pharmaceutical Industries and Associations (EFPIA). Sharing clinical trial information. 2018 [cited 2018 July 30]; Available from: https://www.efpia.eu/about-medicines/development-of-medicines/clinical-trials/sharing-clinical-trialinformation/.

99.Pharmaceutical Research and Manufacturers of America (PhRMA). PhRMA Principles for Responsible Clinical Trial Data Sharing -Certifications. 2017 [cited 2018 July 28]; Available from: https://www.phrma.org/codes-and-guidelines/phrma-principles-forresponsible-clinical-trial-data-sharing-certifications.

100.Vivli. Our members. 2019 [cited 2019 March 1]; Available from: https://vivli.org/members/ourmembers/.

101.Vivli Center for Global Clinical Research Data. 2018 Progress Report. 2019 [cited 2019 April 1]; Available from: https://s3.amazonaws.com/pageturnpro2.com/Publications/201812/4485/87942/PDF/131889563346378000_Vivli_AR_2018.pdf.

102.Chan, A. W., et al., Increasing value and reducing waste: addressing inaccessible research. Lancet, 2014. 383(9913): p. $257-66$.

103.Doshi, P., S. N. Goodman, and J. P. Ioannidis, Raw data from clinical trials: within reach? Trends Pharmacol Sci, 2013. 34(12): p. $645-7$.

104.Gotzsche, P. C., Why we need easy access to all data from all clinical trials and how to accomplish it. Trials, 2011. 12: p. 249. 
105.Ross, J. S. and H. M. Krumholz, Ushering in a new era of open science through data sharing: the wall must come down. JAMA, 2013. 309(13): p. 1355-6.

106.Fecher, B., S. Friesike, and M. Hebing, What drives academic data sharing? PLoS One, 2015. 10(2): p. e0118053.

107.Nimh Collaborative Data Synthesis for Adolescent Depression Trials Study Team including, et al., Advancing Science Through Collaborative Data Sharing and Synthesis. Perspect Psychol Sci, 2013. 8(4): p. 433-44.

108.Sydes, M. R. and D. Ashby, Data Authorship as an Incentive to Data Sharing. N Engl J Med, 2017. 377(4): p. 402.

109.Bierer, B. E., M. Crosas, and H. H. Pierce, Data Authorship as an Incentive to Data Sharing. N Engl J Med, 2017. $377(4)$ : p. 402.

110.Berman, F. and V. Cerf, Science priorities. Who will pay for public access to research data? Science, 2013. 341(6146): p. 616-7.

111.Wilhelm, E. E., E. Oster, and I. Shoulson, Approaches and costs for sharing clinical research data. JAMA, 2014. 311(12): p. 1201-2.

112.Zhu, C. S., et al., Data sharing in clinical trials: An experience with two large cancer screening trials. PLoS Medicine / Public Library of Science, 2017. 14(5): p. e1002304.

113.Naudet, F., et al., Data sharing and reanalysis of randomized controlled trials in leading biomedical journals with a full data sharing policy: Survey of studies published in the BMJ and PLOS Medicine. BMJ (Online), 2018. 360 (no pagination)(k400).

114. Martin Bobrow, et al. Establishing incentives and changing cultures to support data access. 2014 [cited 2019 March 20]; Available from: https://wellcome.ac.uk/sites/default/files/establishing-incentives-and-changing-cultures-to-support-data-accesseagda-may14.pdf.

115.Rockhold, F., P. Nisen, and A. Freeman, Data sharing at a crossroads. New England Journal of Medicine, 2016. 375(12): p. 1115-1117.

116.Kaiser, J., A new portal for patient data. Science, 2018. 361(6399): p. 212.

117.Tucker, K., et al., Protecting patient privacy when sharing patient-level data from clinical trials. BMC Med Res Methodol, 2016. 16 Suppl 1: p. 77.

118.Tudur Smith, C., et al., How should individual participant data (IPD) from publicly funded clinical trials be shared? BMC Medicine, 2015. 13: p. 298.

119.Mazor, K. M., et al., Stakeholders' views on data sharing in multicenter studies. J Comp Eff Res, 2017. 6(6): p. 537-547.

120.van Panhuis, W. G., et al., A systematic review of barriers to data sharing in public health. BMC Public Health, 2014. 14: p. 1144.

121.Bobrow, M., et al. Establishing incentives and changing cultures to support data access. 2014 [cited 2019 January 4]; Available from: https://wellcome.ac.uk/sites/default/files/establishing-incentives-and-changing-cultures-to-support-data-access-eagdamay14.pdf.

122.Bull, S., N. Roberts, and M. Parker, Views of Ethical Best Practices in Sharing Individual-Level Data From Medical and Public Health Research: A Systematic Scoping Review. J Empir Res Hum Res Ethics, 2015. 10(3): p. 225-38.

123.Rowhani-Farid, A., M. Allen, and A. G. Barnett, What incentives increase data sharing in health and medical research? A systematic review. Res Integr Peer Rev, 2017. 2: p. 4.

124.Youngseek Kim, Fostering scientists' data sharing behaviors via data repositories, journal supplements, and personal communication methods. Information Processing and Management, 2017. 53(4): p. 871-885.

125.Hopkins, C., et al., UK publicly funded Clinical Trials Units supported a controlled access approach to share individual participant data but highlighted concerns. Journal of Clinical Epidemiology, 2016. 70: p. 17-25.

Page 17/30 
126.Learned, K., et al., A critical evaluation of genomic data sharing: Barriers to accessing pediatric cancer genomic datasets: $A$ Treehouse Childhood Cancer Initiative experience. Cancer Research. Conference: American Association for Cancer Research Annual Meeting, 2017. 77(13 Supplement 1).

127.Simmonds, M., G. Stewart, and L. Stewart, A decade of individual participant data meta-analyses: A review of current practice. Contemp Clin Trials, 2015. 45(Pt A): p. 76-83.

128.Debray, T. P., et al., Get real in individual participant data (IPD) meta-analysis: a review of the methodology. Res Synth Methods, 2015. 6(4): p. 293-309.

129.Rogozinska, E., et al., Meta-analysis using individual participant data from randomised trials: Opportunities and limitations created by access to raw data. Evidence-Based Medicine, 2017. 22(5): p. 157-162.

130.Stewart, L. A., et al., Preferred Reporting Items for Systematic Review and Meta-Analyses of individual participant data: the PRISMA-IPD Statement. JAMA, 2015. 313(16): p. 1657-65.

131.SAS Institute. SAS Clinical Trial Data Transparency. 2014 [cited 2014 July 20]; Available from: http://support.sas.com/documentation/onlinedoc/clinicaltrial/index.html.

132.SAS Institute, Multi-Sponsor Environment - SAS® Clinical Trial Data Transparency Version 2.3 User Guide. 2018.

133.Clinical Study Data Request. Access to Data 2018 [cited 2018 August 1]; Available from:

https://clinicalstudydatarequest.com/Help/Help-Access-to-Data.aspx.

134.Mospan, G. A. and K. A. Wargo, Researchers' Experience with Clinical Data Sharing. Journal of the American Board of Family Medicine: JABFM, 2016. 29(6): p. 805-807.

135.Mbuagbaw, L., et al., Challenges to complete and useful data sharing. Trials [Electronic Resource], 2017. 18(1): p. 71.

136.Aitken, M., et al., Public responses to the sharing and linkage of health data for research purposes: a systematic review and thematic synthesis of qualitative studies. BMC Med Ethics, 2016. 17(1): p. 73.

137. Hughes, S., et al., Preparing individual patient data from clinical trials for sharing: the GlaxoSmithKline approach. Pharm Stat, 2014. 13(3): p. 179-83.

138. Committee on Strategies for Responsible Sharing of Clinical Trial Data, Board on Health Sciences Policy, and Institute of Medicine. Sharing clinical trial data: maximizing benefits, minimizing risk. Washington (DC): National Academies Press (US); 2015 Apr 20. Appendix B, Concepts and Methods for De-identifying Clinical Trial Data]. Available from:

https://www.ncbi.nIm.nih.gov/books/NBK285994/.

139.Emam, K. E., S. Rodgers, and B. Malin, Anonymising and sharing individual patient data. BMJ (Online), 2015. 350 (no pagination)(h1139).

140.Tierney, J. F., et al., Individual Participant Data (IPD) Meta-analyses of Randomised Controlled Trials: Guidance on Their Use. PLoS Med, 2015. 12(7): p. e1001855.

141.Hollis, S., et al., Best practice for analysis of shared clinical trial data. BMC medical research methodology, 2016. 16(Supplement 1): p. 76.

142.AllTrials. About AllTrials. 2014 [cited 2018 August 1]; Available from: http://www.alltrials.net/find-out-more/about-alltrials/. 143.GlaxoSmithKline. Data transparency. 2014 [cited 2018 August 1]; Available from: https://www.gsk.com/en-gb/behind-thescience/innovation/data-transparency/.

144.Simmonds, M. C., et al., Safety and effectiveness of recombinant human bone morphogenetic protein-2 for spinal fusion: a meta-analysis of individual-participant data. Ann Intern Med, 2013. 158(12): p. 877-89.

Page $18 / 30$ 
145.Fu, R., et al., Effectiveness and harms of recombinant human bone morphogenetic protein-2 in spine fusion: a systematic review and meta-analysis. Ann Intern Med, 2013. 158(12): p. 890-902.

146.Krumholz, H. M., et al., A historic moment for open science: the Yale University Open Data Access project and medtronic. Ann Intern Med, 2013. 158(12): p. 910-1.

147. Hrobjartsson, A., Why did it take 19 months to retrieve clinical trial data from a non-profit organisation? BMJ, 2013. 347: p. f6927.

148.Savage, C. J. and A. J. Vickers, Empirical study of data sharing by authors publishing in PLoS journals. PLoS One, 2009. 4(9): p. e7078.

149.Filippon, J., Slow and costly access to anonymised patient data impedes academic research. BMJ (Online), 2015.351 (no pagination)(h5087).

150.Tudur Smith, C., et al., Sharing individual participant data from clinical trials: an opinion survey regarding the establishment of a central repository. PLoS One, 2014. 9(5): p. e97886.

151.Berlin, J. A., et al., Bumps and bridges on the road to responsible sharing of clinical trial data. Clin Trials, 2014. 11(1): p. 7-12.

152.Huser, V. and D. Shmueli-Blumberg, Data sharing platforms for de-identified data from human clinical trials. Clinical Trials, 2018. 15(4): p. 413-423.

153.So, D. and B. M. Knoppers, Ethics approval in applications for open-access clinical trial data: An analysis of researcher statements to clinicalstudydatarequest.com. PLoS ONE [Electronic Resource], 2017. 12(9): p. e0184491.

154.Sydes, M. R., et al., Sharing data from clinical trials: The rationale for a controlled access approach. Trials, 2015.16 (1) (no pagination)(104).

155.Green, A. K., et al., The project data sphere initiative: Accelerating cancer research by sharing data. Oncologist, 2015. 20(5): p. 464-472.

156.Eichler, H. G. and F. Sweeney, The evolution of clinical trials: Can we address the challenges of the future? Clinical Trials, 2018. 15(1_suppl): p. 27-32.

157.Khusro, A. and C. Aarti, TB-PACTS: A fresh emphatic data sharing approach. Asian Pacific Journal of Tropical Disease, 2017. 7(2): p. 97-98.

158.Bertagnolli, M. M., et al., Advantages of a Truly Open-Access Data-Sharing Model. New England Journal of Medicine, 2017. 376(12): p. 1178-1181.

159.Asare, A. L., et al., Clinical trial data access: Opening doors with TrialShare. Journal of Allergy and Clinical Immunology, 2016. 138(3): p. 724-726.

160.Zinner, D. E., G. Pham-Kanter, and E. G. Campbell, The Changing Nature of Scientific Sharing and Withholding in Academic Life Sciences Research: Trends From National Surveys in 2000 and 2013. Acad Med, 2016. 91(3): p. 433-40.

161.Rowhani-Farid, A. and A. G. Barnett, Has open data arrived at the British Medical Journal (BMJ)? An observational study. BMJ Open, 2016. 6(10): p. e011784.

162.Strom, B. L., et al., Data Sharing - Is the Juice Worth the Squeeze? New England Journal of Medicine, 2016. 375(17): p. 16081609.

163.Bonini, S., et al., Transparency and the European Medicines Agency-sharing of clinical trial data. N Engl J Med, 2014. 371(26): p. 2452-5. 
164.Drazen, J. M., Sharing individual patient data from clinical trials. N Engl J Med, 2015. 372(3): p. 201-2.

165.Lo, B., Sharing Clinical Trial Data: Maximizing Benefits, Minimizing Risk. JAMA, 2015.

166.International Consortium of Investigators for Fairness in Trial Data, S., et al., Toward Fairness in Data Sharing. N Engl J Med, 2016. 375(5): p. 405-7.

167.Veroniki, A. A., et al., Contacting authors to retrieve individual patient data: study protocol for a randomized controlled trial. Trials, 2016. 17(1): p. 138.

168.Navar, A.M., M. J. Pencina, and E. D. Peterson, Open Access Platforms for Sharing Clinical Trial Data-Reply. JAMA, 2016. 316(6): p. 666-7.

169.Ross, J. S. and H. M. Krumholz, Open Access Platforms for Sharing Clinical Trial Data. JAMA, 2016. 316(6): p. 666.

170.Navar, A.M., et al., Use of Open Access Platforms for Clinical Trial Data. JAMA, 2016. 315(12): p. 1283-4.

171.Garrison, N. A., et al., A systematic literature review of individuals' perspectives on broad consent and data sharing in the United States. Genet Med, 2016. 18(7): p. 663-71.

172.Clarke, M. J., Individual patient data meta-analyses. Best Practice and Research: Clinical Obstetrics and Gynaecology, 2005. 19(1 SPEC. ISS.): p. 47-55.

173.Jaspers, G. J. and P. L. J. Degraeuwe, A failed attempt to conduct an individual patient data meta-analysis. Systematic Reviews, 2014. 3 (1) (no pagination)(97).

174. Tierney, J. F., et al., Individual participant data (IPD) metaanalyses of randomised controlled trials: Uidance on their use. PLoS Medicine, 2015. 12(7).

175.Veroniki, A. A., et al., Contacting authors to retrieve individual patient data: Study protocol for a randomized controlled trial. Trials, 2016. 17 (1) (no pagination)(138).

176.Clinical Study Data Request. Sponsor Specific Details: Astellas. 2019 [cited 2019 March 1]; Available from: https://www.clinicalstudydatarequest.com/Study-Sponsors/Study-Sponsors-Astellas.aspx.

177.Chugai Pharmaceutical Co. Ltd. Clinical trial data sharing. 2018 [cited 2019 March 12]; Available from: https://www.chugaipharm.co.jp/english/profile/rd/guideline/ctds_request.html.

178.Nisen, P. and F. Rockhold, Access to patient-level data from GlaxoSmithKline clinical trials. N Engl J Med, 2013. 369(5): p. 4758.

179.GlaxoSmithKline. Data transparency. 2014 [cited 2019 March 12]; Available from: https://www.gsk.com/en-gb/research/ourapproach/trials-in-people/data-transparency/.

180.Novartis. Novartis Position on Clinical Study Transparency-Clinical Study Registration, Results Reporting and Data Sharing. 2016 [cited 20189 March 12]; Available from: https://www.novartis.com/sites/www.novartis.com/files/clinical-trial-datatransparency.pdf.

181.Sumitomo Dainippon Pharma. Disclosure of Clinical Study Data. 2018 [cited 2019 March 13]; Available from: https://www.dspharma.com/rd/clinical/clinical_study_data.html.

182. Yale University Open Data Access (YODA) Project. How to request data. [cited 2019 March 13]; Available from: https://yoda.yale.edu/how-request-data.

183.Johnson \& Johnson. Johnson \& Johnson Announces Clinical Trial Data Sharing Agreement with Yale School of Medicine. 2014 [cited 2014 July 1]; Available from: http://www.jnj.com/news/all/johnson-and-johnson-announces-clinical-trial-data-sharing-

Page 20/30 
agreement-with-yale-school-of-medicine.

184. Harlan M. Krumholz. Give the data to the people. 2014 [cited 2014 July 4]; Available from: http://www.nytimes.com/2014/02/03/opinion/give-the-data-to-the-people.html?_r = 0.

185.Vivli. Aegerion Pharmaceuticals. 2019 [cited 2019 March 1]; Available from: https://vivli.org/ourmember/aegerionpharmaceuticals/.

186.Biogen. Biogen clinical trial transparency and data sharing policy. 2019 [cited 2019 March 5]; Available from: http://clinicalresearch.biogen.com/.

187.Daiichi-Sankyo. Clinical Trial Information Disclosure. 2018 [cited 2018 July 20]; Available from: https://www.daiichisankyo.com/rd/our_approach/clinical_studies/index.html.

188.Pfizer. Clinical Trials Data Access - Independent Review Panel Charter. 2014 [cited 2019 March 21]; Available from: https://www.pfizer.com/files/research/research_clinical_trials/Independent_Review_Panel_Charter_01312014.pdf.

189.AstraZeneca. AstraZeneca Group of Companies-Data Request Portal. 2018 [cited 2018 July 20]; Available from: https://astrazenecagroup-dt.pharmacm.com/DT/Home.

190.Natalie Andrews. AstraZeneca Clinical Trial Transparency and External Sharing of Individual Patient-Level Data. 2015 [cited 2018 July 20]; Available from: https://www.phusewiki.org/docs/Conference\%202015\%20PP\%20Papers/PP17.pdf.

191.AstraZeneca. Disclosure Commitment. 2019 [cited 2019 March 21]; Available from:

https://astrazenecagrouptrials.pharmacm.com/ST/Submission/Disclosure.

192.Pencina, M. J., et al., Supporting open access to clinical trial data for researchers: The Duke Clinical Research Institute-BristolMyers Squibb Supporting Open Access to Researchers Initiative. American Heart Journal, 2016. 172: p. 64-69.

193.Celgene. Clinical trial data sharing policy. 2017 [cited 2019 March 14]; Available from:

https://media.celgene.com/content/uploads/clinical-trial-data-sharing-policy.pdf.

194.Chiesi Group. Clinical trial transparency statement. 2018 [cited 2019 March 21]; Available from:

https://www.chiesi.com/en/research-and-development/clinical-trial-transparency-and-data-sharing/.

195.EMD Serono. Responsible data sharing instruction document. [cited 2019 April 18]; Available from:

https://www.emdgroup.com/content/dam/web/corporate/non-images/business-

specifics/healthcare/US/Responsible_Data_Sharing_Instruction_Document_US.pdf.

196.The Menarini Group. User Registration. 2018 [cited 2018 July 30]; Available from: https://clinicaltrials.menarini.com/enUS/Home/Datasharing.

197.The Menarini Group. Datasharing. 2018 [cited 2019 March 21]; Available from: https://clinicaltrials.menarini.com/enUS/Home/Datasharing.

198.Servier. Data request portal. 2018 [cited 2019 March 21]; Available from: https://clinicaltrials.servier.com/data-request-portal/.

199.Almirall. Clinical trials. 2019 [cited 2019 March 14]; Available from: https://www.almirall.com/en/about-almirall/research-anddevelopment/clinical-trials.

200.Amgen. Clinical Trial Data Sharing Request 2019 [cited 2019 March 14]; Available from: https://www.amgen.com/about/howwe-operate/policies-practices-and-disclosures/ethical-research/clinical-data-transparency-practices/clinical-trial-data-sharingrequest/.

201.Bial. Transparency. 2014 [cited 2019 March 14]; Available from: https://www.bial.com/en/r_d.2/transparency.208/transparency.a484.html.

Page $21 / 30$ 
202.Esteve. Commitment letter. 2014 [cited 2019 March 14]; Available from: https://www.efpia.eu/media/25446/esteve.pdf.

203.Grunenthal. Clinical data sharing with researchers. 2018 [cited 2019 March 21]; Available from: https://www.grunenthal.com/rd-vision-mission/clinical-trials/data-sharing-clinical-trials.

204.Ipsen. Transparency and trust. 2019 [cited 2019 March 14]; Available from:

https://www.ipsen.com/commitments/transparency-and-trust/clinical-trials/.

205.Leo Pharma. LEO Pharma's Position on Public Access to Clinical Trials Information. [cited 2019 March 14]; Available from: http://www.leo-pharma.com/Home/Research-and-Development/Clinical-trial-disclosure/LEO-Pharmas-position-ontransparency.aspx.

206.Leo Pharma. How to get access to anonymised patient-level data. [cited 2019 March 14]; Available from: http://www.leopharma.com/Home/Research-and-Development/Clinical-trial-disclosure/Access-to-patient-level-

data.aspx\#Step_1:_Fasibility_assessment_by_LEO_Pharma.

207.Lundbeck. Policy for scientific publications and responsible clinical trial data sharing. 2018 [cited 2019 March 14]; Available from: https://www.lundbeck.com/global/brain-disorders/research-and-development/policy-for-scientific-publications-andresponsible-clinical-trial-data-sharing/clinical-data-sharing.

208.Otsuka Pharmaceutical Co. Ltd. For researchers: Overview of researcher access to patient-level data 2019 [cited 2019 March 14]; Available from: https://clinical-trials.otsuka.com/For-Researchers.aspx.

209.Novo Nordisk. How to access clinical trial datasets. [cited 2019 March 14]; Available from: https://www.novonordisktrials.com/how-access-clinical-trial-datasets.

210.Novo Nordisk. Independent review board charter. [cited 2019 March 14]; Available from: https://www.novonordisktrials.com/sites/novonordisk/themes/novo/img/document/Independent\%20Review\%20Board\%20Charter.pdf.

211.Orion Corporation. Ethics in the Clinical Research Phases. [cited 2019 March 14]; Available from: https://www.orion.fi/en/Oriongroup/Sustainability/policies/pharmaceutical-rd-ethics-policy/ethics-in-clinical-research/.

212.Vifor Pharma. Responsibility highlights report 20172018 [cited 2019 March 14]; Available from:

http://www.viforpharma.com/ /media/Files/V/Vifor-Pharma/documents/en/responsibility/Vifor_Pharma_CSR_Report_2017.pdf.

213.Clinical Study Data Request. Independent review panel charter. [cited 2018 August 1]; Available from:

https://clinicalstudydatarequest.com/Documents/Independent_Review_Panel_Charter.pdf.

214.Yale University Open Data Access (YODA) Project. Procedures to Guide External Investigator Access to Clinical Trial Data. 2016 May 2016 [cited 2018 August 1]; Available from:

http://yoda.yale.edu/sites/default/files/files/YODA\%20Project\%20Data\%20Release\%20Procedures\%20June\%202016.pdf.

215.Duke Clinical Research Institute. Bristol-Myers Squibb data sharing independent review committee (IRC) charter. 2017 [cited 2019 March 21]; Available from: https://dcri.org/wp-content/uploads/2017/10/BMS_Charter_FINAL-approved-by-IRC-10.13.171.pdf.

216.Vivli. Biogen. 2019 [cited 2019 March 21]; Available from: https://vivli.org/ourmember/biogen/.

217.Grunenthal. Requesting access to clinical data. 2018 [cited 2019 March 21]; Available from:

https://www.grunenthal.com/-/media/projects/corporatewebsite/com/pdf/content_clincialtrial-portal/2018-11-02_sdn-pubdisclaccess_requesting-access-to-clinical-data.pdf?la = en\&hash = 2D683A66BEA23C98044D2833D732713B581E2929.

218.EMD Serono. Summary of EMD Serono's responsible data sharing policy. [cited 2019 March 22].

\section{Tables}

Page 22/30 
Table 1. Included articles with direct relevance to guide researchers in the conduct of IPDMA.

\section{Study}

Abo-Zaid et al. (2012). "Individual participant data metaanalysis of prognostic factor studies: state of the art?" [ 22]

Berlin et al. (2014). "Bumps and bridges on the road to responsible sharing of clinical trial data." [ 151]

Clarke (2005). "Individual patient data meta-analyses." [ 172]

Higgins and Green. "Cochrane Handbook for Systematic Reviews of Interventions Version 5.1.0 [updated March 2011]." [ 14]

Huang, et al. (2014). "Distribution and epidemiological characteristics of published individual patient data metaanalyses." [ 18]

Jaspers and Degraeuwe (2014). "A failed attempt to conduct an individual patient data meta-analysis." [ 173]

Nevitt et al. (2017). "Exploring changes over time and characteristics associated with data retrieval across individual participant data meta-analyses: Systematic review." [ 17]

Polanin (2018). "Efforts to retrieve individual participant data sets for use in a meta-analysis result in moderate data sharing but many data sets remain missing." [ 81]

Polanin and Terzian (2019). "A data-sharing agreement helps to increase researchers' willingness to share primary data: results from a randomized controlled trial." [ 83]

Polanin and Williams (2016). "Overcoming obstacles in obtaining individual participant data for meta-analysis." [ 80]

Riley et al. (2010). "Meta-analysis of individual participant data: rationale, conduct, and reporting." [21]

Ross (2016). "Clinical research data sharing: what an open science world means for researchers involved in evidence synthesis." [ 26]

Stewart and Clarke (1995). "Practical methodology of meta-analyses (overviews) using updated individual patient data. Cochrane Working Group." [ 6]

Tierney et al. (2015). "Individual Participant Data (IPD) Meta-analyses of Randomised Controlled Trials: Guidance on Their Use." [ 174]

Veroniki et al. (2016). "Contacting authors to retrieve individual patient data: Study protocol for a randomized controlled trial." [ 175]

Young and Hopewell (2011). "Methods for obtaining unpublished data." [ 79]
Description

Systematic review of IPDMAs of prognostic factors aimed at describing the conduct, evaluation and commonly experienced challenges.

Literature review providing guidance on the process of obtaining and combining datasets from different sources.

Systematic review describing the rationale of IPDMA and processes for obtaining IPD.

The Cochrane Handbook provides guidance to authors performing Cochrane Intervention reviews. Chapter 18 describes IPDMAs, including the collaboration process.

Survey of published IPDMAs until August 2012 describing their distribution and epidemiologic characteristics.

Case report describing the process of pursuing data and lessons learned from an IPDMA, which could not be completed.

Systematic review of IPDMAs conducted until August 2015, which identifies study factors significantly associated with obtaining a high proportion of IPD.

Meta-analysis of IPDMAs, which examines the success rate of obtaining IPD solely through direct contact with study authors.

Randomized controlled trial assessing the effect of IPDMA authors providing a data sharing agreement on primary author data sharing.

Review, that provides solutions to barriers encountered while obtaining IPD for IPDMA.

Description of rationale, conduct and reporting standards of IPDMA, which also describes recent trends in published IPDMA.

Commentary on general data sharing trends and predictions, including some barriers to identifying, obtaining and combining datasets for IPDMA.

The first practical guide describing IPDMA conduct, including discussion of planning, obtaining and analyzing IPD.

An updated guide describing IPDMA conduct, including discussion of planning, obtaining and analyzing IPD.

Study protocol for a randomized controlled trial comparing data acquisition techniques.

Review of studies, that examines techniques for obtaining IPD by contacting primary study authors.

Table 2. Approach to email correspondence 


\begin{tabular}{|l|}
\hline Suggestion \\
\hline Corresponding authors are typically the first point of contact when requesting study data via email \\
\hline $\begin{array}{l}\text { When possible, send emails on behalf of a well-known research organization, from someone with professional } \\
\text { authority or from a personal acquaintance }\end{array}$ \\
\hline Include the primary investigator, research coordinator and key team members in requesting emails \\
\hline Include obvious keywords in the subject line allowing easy message retrieval \\
\hline Clearly define a purpose and exclude use of acronyms as well as emotional cues \\
\hline Express concern for alternative duties and avoid rude, irritating, or unprofessional language \\
\hline Describe recognition for data sharing \\
\hline Request a teleconference or in-person meeting to discuss several issues in a brief period \\
\hline Attach a study protocol and other important documents to requesting emails \\
\hline For each study, generate a list of contacts and corresponding responsibilities \\
\hline
\end{tabular}

Table 3. Data availability of pharmaceutical companies displaying certification via PhRMA or EFPIA websites which solicit data requests via online data sharing platform [ $47,98,99]$ 
Request point: https://clinicalstudydatarequest.com [ 52]

Astellas [ 55, 176] Phase 1, 2, 3 and 4 studies for indications which have been approved by the US and, or EU

Bayer [ 56] All trials required for regulatory approval

Boehringer Ingelheim [ 58] All trials with published results

Chugai [ 177] All sponsored clinical trials

Eisai [ 61] Phase 2, 3 and 4 studies required for regulatory approval which have been approved by the US and, or EU.

Elli Lilly and Company [ 62] Phase 2, 3 and 4 studies required for regulatory approval

January 1, 2010

January 1, 2014

January 1, 1998

January 1, 2014

January 1, 2014

January 1, 1999

Phase 2, 3, 4 global studies after January 2007

Phase 2, 3, 4 regional/global studies for drugs approved in US and EU since January 1, 2014

GlaxoSmithKline [ 64, 178, All global interventional studies

December 1, 2000

179]

All interventional studies since 2013

Other studies where data are provided to researchers

Novartis [ 180]

Phase 2 and 3 studies required for regulatory approval in the EU or US

January 1, 2014

Roche [ 75]

Requested studies must support the indication

Sanofi [67]

All phase 2 and 3 studies or phase 4 studies required for regulatory approval. Products

January 1, 1999 terminated from development.

Sumitomo Dainippon All trials, for approved indications, required for regulatory approval in the US and EU

January 1, 2014

Phase 2, 3, and 4 interventional clinical studies included in the submission package for

January 1, 2014

Pharma Co, Ltd [ 181] approved medications in the US, EU, or Japan

Takeda [69]

Phase 1,2,3 and 4 trials which support approved products and products terminated from development

Phase 2, 3, and 4 study data for approved medicines and indications

January 1, 2005

UCB [ 70]

Phase 2, 3, and 4 study data for approved medications

November 1, 2008

Viiv Healthcare [ 65]

Phase 1, 2, 3, and 4 interventional clinical studies

Phase 1, 2, 3, and 4 interventional clinical studies for terminated compounds

November 1, 2017

January 1, 2010

Request point: http://yoda.yale.edu/how- request-data [ 182]

Johnson and All clinical research data

January $1,1990^{2}$

Johnson/Janssen [ 72, 183,

184]

Request point: https://vivli.org ${ }^{3}$ [ 100]

Abbvie [ 53] Phase 2, 3 and 4 interventional clinical studies for medicinal products and indications which received authorization in US or EU

Aegerion Pharmaceuticals [ Anonymized, patient-level and study-level clinical trial data and other information (such Unclear

185] as protocols and clinical study reports) where available

Biogen [ 186] Phase 1, 2, 3 and 4 interventional clinical trials for products and indications submitted to and approved in the US and EU.

Boehringer Ingelheim [ 58] All trials with published results

Daiichi-Sankyo [ 187] Phase 2, 3 and 4 interventional clinical studies submitted for approved medications in January 1, 2014 US, EU or Japan

GlaxoSmithKline [ 64, 179] Global interventional studies

December 1, 2000

Interventional studies evaluating medicines, starting in or after 2013

Consumer healthcare studies completed on or after January 1, 2018

Pfizer [ 66, 188] Global interventional studies conducted for medicines, vaccines, and medical devices

September 1, 2007 which were terminated or are approved in the US or EU

Takeda [ 69] Phase 1,2,3 and 4 trials which support approved products and products terminated from development

Request point: https://astrazenecagroup-dt.pharmacm.com/DT/Home/Login

AstraZeneca [ 189-191] $\quad$ Phase 1, 2, 3, or 4 studies for approved indications in the US, EU, or Japan January 1, 2009*

Request point: https://biogen-dt-external.pharmacm.com/DT/Home

Biogen [ 57] Phase 1, 2, 3, or 4 studies for discontinued compounds or those approved in the US and EU

Request point: https://fasttrack.bms.com/

Bristol-Myers Squibb [ 59, Phase 1, 2, 3, or 4 study data for medicines and indications approved in the US or EU

192]

Request point: https://www.celgeneclinicaldatasharing.com

Celgene [ 60, 193] Study data for compounds and indications approved in the US and EU

Request point: http://www.chiesi.com/en/chiesi-clinical-trial-data-request-portal/

Chiesi [ 194] Study data for medications approved by the FDA or EMA

January 1, 2014

Request point: https://www.emdgroup.com/en/research/our-approach-to-research-and-development/healthcare/clinical-trials/commitment- 
responsible-data-sharing.html

EMD Serono [ 63, 195] Study data for products and indications approved in the US and EU

January 1, 2014

Request point: https://clinicaltrials.menarini.com/en-US/Home/Register

Menarini [ 196, 197] Study data for medications and indications approved in the US and EU Unclear

Request point: http://engaggezone.msd.com/

Merck \& Co. [ 73] $\quad$ Study data submit for regulatory approval in the US and EU for approved indications

September 1, 2007

Request point: purduepharma.com/healthcare-professionals/clinical-trials/request-trial-data

Purdue Pharmaceuticals [ Phase 2, 3 or 4 study data for drug products and their approved uses in the US for 74] approved indications

January 1, 2014

Request point: https://clinicaltrials.servier.com/data-request-portal/login/

Servier [ 198] $\quad$ Study data for approved medications or indications in European Economic Area or US

January 1, 2014

1 Funders include the Bill and Melinda Gates Foundation, Cancer Research UK, Medical Research Council, and Wellcome Trust.

2 Electronic data is available as far back as 1990. Study data prior to 1990 are only available in paper format and are not readily accessible.

3 Vivli.org also provides access to data sponsored, stored or generated by BioLINCC, Critical Path Institute, Doris Duke Charitable Foundation, Duke University, Harvard University, ImmPort, Johns Hopkins University, Project Data Sphere, the Leona M. and Harry B. Helmsley Charitable Trust and the University of California San Francisco.

Table 4. Pharmaceutical companies displaying certification via PhRMA or EFPIA websites, which solicit data requests through email [47, 98, 99]

\begin{tabular}{|c|c|c|}
\hline $\begin{array}{l}\text { Pharmaceutical company and } \\
\text { access point }\end{array}$ & Advertised trial data made available & $\begin{array}{l}\text { Publicized date of earliest } \\
\text { available data }\end{array}$ \\
\hline Almirall [ 199] & Study data for approved medications and indications in the US or & January 1, 2014 \\
\hline R\&D@almirall.com & EU & \\
\hline $\begin{array}{l}\text { Amgen [ 54, 200] } \\
\text { datasharing@Amgen.com }\end{array}$ & $\begin{array}{l}\text { Study data submit for regulatory approval in the US and EU for } \\
\text { approved indications }\end{array}$ & Unclear \\
\hline $\begin{array}{l}\text { Bial [ 201] } \\
\text { clinical.trials@bial.com }\end{array}$ & $\begin{array}{l}\text { Study data for approved medications and indications in the US or } \\
\text { EU }\end{array}$ & Unclear \\
\hline Esteve [ 202] & Unclear & Unclear \\
\hline $\begin{array}{l}\text { Grunenthal [ 203] } \\
\text { clinicaltrialportal@grunenthal.com }\end{array}$ & $\begin{array}{l}\text { Study data submit in support of licensed treatments in the US or } \\
\text { EU }\end{array}$ & July 15, 2014 \\
\hline $\begin{array}{l}\text { Ipsen [ } 204] \\
\text { Unclear request point }\end{array}$ & Unclear & Unclear \\
\hline $\begin{array}{l}\text { Leo Pharma [ 205, 206] } \\
\text { disclosure@leo-pharma.com }\end{array}$ & $\begin{array}{l}\text { Study data for any approved product or products from discontinued } \\
\text { trials which began since } 2014\end{array}$ & January 1,2000 \\
\hline $\begin{array}{l}\text { Lundbeck [ 207] } \\
\text { clinicaldataaccess@lundbeck.com }\end{array}$ & $\begin{array}{l}\text { Study data for approved medications and indications in the US or } \\
\text { EU }\end{array}$ & January 1, 2014 \\
\hline $\begin{array}{l}\text { Otsuka [ 208] } \\
\text { DT-inquiry@otsuka.jp }\end{array}$ & Study data submit to the US or EU for regulatory approval & January 1, 2014 \\
\hline $\begin{array}{l}\text { Novo Nordisk [ 209, 210] } \\
\text { irb-secretariat@novonordisk.com }\end{array}$ & Study data for product indications approved in the US and EU & January 1, 2001 \\
\hline $\begin{array}{l}\text { Orion Pharma [ 211] } \\
\text { Unclear request point }\end{array}$ & $\begin{array}{l}\text { Study data for medications which have obtained received market } \\
\text { authorisation }\end{array}$ & Unclear \\
\hline $\begin{array}{l}\text { Vifor pharma [ } 212] \\
\text { Unclear request point }\end{array}$ & $\begin{array}{l}\text { Provide academic researchers access to clinical trial data upon } \\
\text { request }\end{array}$ & Unclear \\
\hline $\begin{array}{l}\text { Shire [ 68] } \\
\text { clinicaltrialdata@shire.com }\end{array}$ & $\begin{array}{l}\text { Study data for compounds and indications approved in the US and } \\
\text { EU }\end{array}$ & January 1, 2014 \\
\hline
\end{tabular}


Table 5. Data request review process of pharmaceutical companies displaying certification via PhRMA or EFPIA websites which solicit data requests via online data sharing platform [ $47,98,99]$ 
Clinical Study Research Proposals are checked and reviewed in 3 stages: Stage 1, by the Wellcome Trust which is the secretariat for Data Request the Independent Review Panel (IRP) ; Stage 2, by the study Sponsors/Funders; Stage 3, by the IRP.

affiliates $^{1}$ [

89, 213]

Janssen [ 214]

During the Review, the YODA Project will evaluate submitted requests and associated registration materials to ensure that all required information has been provided.

All requests for data will undergo review upon receipt by the YODA Project. During this review, the YODA Project will evaluate submitted requests and associated registration materials to ensure that all required information has been provided and that the Research Proposal has scientific merit.

Requests will undergo External Review if the YODA Project is unable to verify the scientific merit of the Research Proposal.

Bristol-Myers In Review: The request/proposal is currently being reviewed internally by a qualified panel of Bristol-Myers Squibb Squibb [ 59, experts. If the proposal is considered within scope, the request will undergo an additional review by the independent 215] review committee (DCRI).

Pfizer [ 66, Purpose of Independent Review Panel An internal Pfizer Review Committee conducts the initial review of in scope 188] requests. Any request approved by Pfizer will not require a secondary review by the Independent Review Panel. Pfizer is piloting the use of an Independent Review Panel during 2014. The Panel will review any proposal declined, or partially approved, by Pfizer. The role of the Panel is to review the application, the rationale for Pfizer's response, and to make a final decision. The decision of the Panel will be binding.

Purdue The Purdue Scientific Review Board (SRB) will adjudicate all requests for Information. The SRB will consist of Purdue Pharma [ 74] employees selected by the Chief Medical Officer (CMO) from relevant departments, such as Research and Development, Medical Affairs, Law, and Ethics \& Compliance, and two researchers or external experts who are not employees of Purdue.

Biogen [ 57, Biogen reviews all data requests internally based on the criteria set forth in our Clinical Trial Transparency and Data 216] Sharing Policy. Requests that are denied in whole or in part are then sent to an independent external review body, whose decision will be made transparent.

AstraZeneca [ An independent Scientific Review Board to review and approve requests. The Scientific Review Board will review 191] requests that go back as far as 2009 through this process. All other requests for data beyond that will continue to be reviewed by AstraZeneca on a case-by-case basis.

Menarini All requests will be reviewed internally by a qualified panel of Menarini Group experts (Scientific Secretariat) and then Group [ 197] passed to an Independent Review Committee (IRC) of external experts for further review.

Servier [ 198] Servier will conduct the initial review, including scientific qualification of the researcher, the robustness and scientific merit of the research proposal, the ability of the requested data to answer the research question, and the technical feasibility. If Servier partially approves or declines the request, we send our decision to the IRB for review. The decision made by the IRB is final and binding for Servier.

Abbvie [ 53] All requests from qualified researchers for access to AbbVie clinical data and information will be managed by Vivli and AbbVie. In cases where we reject a particular request based on scientific merit, the request, along with the record of our denial of the request, shall be forwarded to the Access to Clinical Research Information Board (ATCRIB) for a final decision, according to the ATCRIB charter. The ATCRIB is composed of scientists and/or health care professionals who are not AbbVie employees.

Almirall [ $\quad$ All requests will be evaluated independently on a case-by-case.

199]

Amgen [ 54, Research proposals will be reviewed by a committee of internal advisors. For clinical trials that are subject to 200] agreements with co-development partners, Amgen will liaise with the applicable partners regarding any data sharing requests. In general, Amgen does not support external research questions that involve access to individual patient level data for the purpose of re-evaluating safety and efficacy issues already addressed in the product labelling. If the outcome of the internal review is to decline the request, a Data Sharing Independent Review Panel will arbitrate and make the final decision.

Bial [ 201] Each request will be evaluated by an independent Scientific Review Board and will be based on criteria that balance the need for scientific development with the need to protect patient privacy.

Chiesi [ 194] An appointed Chiesi Evaluation Committee starts the assessment of the research proposal. In case of a negative evaluation, but no direct competition is envisaged, Chiesi forwards the assessment to a Scientific Review Board, composed by qualified researchers who are not Chiesi employees.

Esteve [ 202] $\quad$ Unable to locate

Grunenthal [ $\quad$ Requests for access to clinical data will be subject to assessment and approval by a Grünenthal Board and then by an 217] independent Scientific Review Board.

Ipsen [ 204] Unable to locate. 
Leo Pharma [ The evaluation of the data request and the decision on access to data is made by the external Patient and Scientific

205, 206] Review Board. The Patient and Scientific Review Board comprise three highly experienced scientists while two seats are allocated to representatives of patient associations. The decision by the Patient and Scientific Review Board is made independently of LEO Pharma.

Lundbeck [ An external scientific review board is responsible for assessing and granting requests from qualified scientific and 207] medical researchers. If the scientific review board rejects a request, the scientific review board can advise a resubmission.

Otsuka [ 208] $\quad$ Research proposals requesting patient-level data are reviewed by an Independent Review Panel at Western Institutional Review Board Copernicus Group. Research proposals for non-listed studies are examined on a case-by-case basis by Otsuka in consultation with the Independent Review Panel.

Celgene [ 60, A group of individuals selected by the Celgene Clinical Trial Data Sharing Steering Committee composed of external

193] experts to provide an unbiased review of research proposals submitted by researchers to ensure that the proposals are robust, scientifically sound with a valid and clearly defined hypothesis and include both an analysis and publication plan.

Novo The Independent Review Board assesses all complete requests and approves or rejects the proposal without any Nordisk [ interference from Novo Nordisk.

209, 210]

Orion After a marketing authorisation has been granted to our new drug, we allow access to our patient-level data based on a Pharma [ scientific review of the request and the proposal from the external research group consisting of qualified scientific and 211] medical researchers.

Vifor Unable to locate.

pharma [

212]

Shire [ 68] Once Shire assesses the validity of the researcher's data request and determines appropriate consent(s) exists for requested product(s) and indication(s), an internal team made of subject matter experts will review the eligibility of the proposed research against the criteria below and render a decision. In cases where the validity of the researcher or proposed request is in question, Shire will defer the request to an external Independent Review Panel for a final, objective opinion.

EMD Serono [ Researchers' requests will be evaluated initially by an internal committee at EMD Serono, which may decide to approve

63, 218] the request. If the EMD Serono committee denies the request, the request will be escalated to the EMD Serono Scientific Review Board for a second review (de novo). The Board shall include scientists and/or healthcare professionals who are not employees of EMD Serono.

Merck \& $\quad$ Completed applications will be reviewed by MSD with Input as needed from an External Scientific Review Board

Co. [ 73] comprised of non-MSD scientists or physicians.

1 Industry affiliates include Astellas, Bayer, Boehringer Ingelheim, Chugai, Eisai, GlaxoSmithKline, Lilly, Novartis, Roche, Sanofi, Sumitomo Dainippon, Takeda, UCB, and Viiv healthcare. Non-industry affiliates include Bill and Melinda Gates Foundation, Cancer Research UK, Medical Research Council, and Wellcome Trust.

\section{Figures}




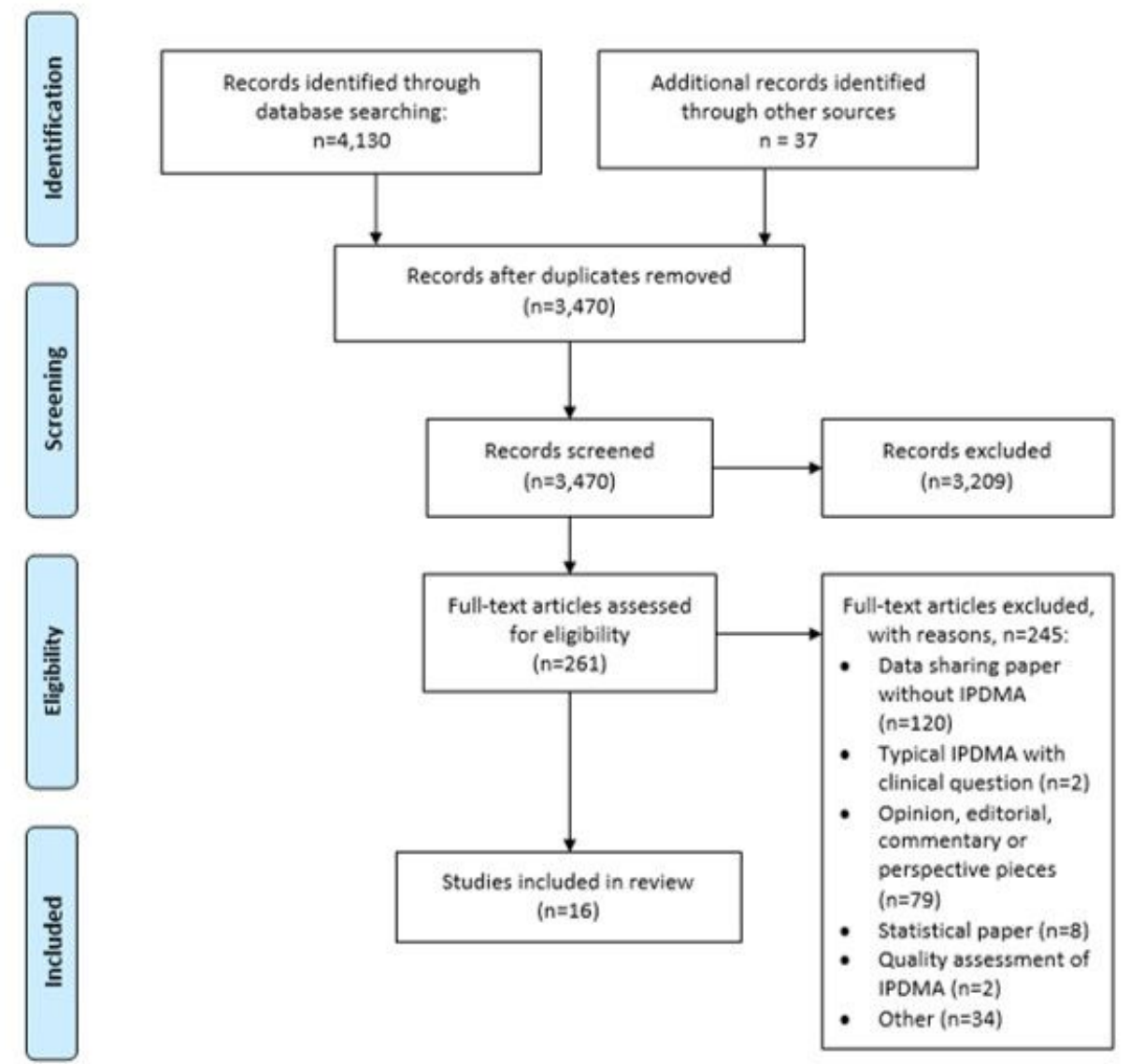

Figure 1

PRISMA flow diagram

\section{Supplementary Files}

This is a list of supplementary files associated with this preprint. Click to download.

- MethodspaperappendixAugust12.docx 